\title{
Low-Complexity Detection Based on Landweber Method in the Uplink of Massive MIMO Systems
}

\author{
Wence Zhang, Xu Bao, Jisheng Dai \\ Jiangsu University, China \\ Emails: \{wencezhang,xbao,jsdai\}@ujs.edu.cn
}

\begin{abstract}
In this paper, we present low-complexity uplink detection algorithms in Massive MIMO systems. We treat the uplink detection as an ill-posed problem and adopt Landweber Method to solve it. In order to reduce the computational complexity and increase the convergence rate, we optimize the relax factor and propose improved Landweber Method with optimal relax factor (ILM-O) algorithm. We also try to reduce the order of Landweber Method by introducing a set of coefficients and propose reduced order Landweber Method (ROLM) algorithm. A analysis on the convergence and the complexity is provided. Numerical results show that the proposed algorithms outperform the existing algorithm significantly when the system scale is large.
\end{abstract}

\section{INTRODUCTION}

Mobile communications systems have undergone tremendous development during the past decades. One of the key technology in the air interface that has boosted the development of mobile communication systems is Multiple-InputMultiple-Output (MIMO) systems. By using multiple antennas, MIMO systems can significantly improve the data rates and reliability of the communication links [1]. As an enhanced version of MIMO, Massive MIMO systems which use tens to hundreds of antennas at the base station (BS) can provide excess degree of systems and improve both the spectrum efficiency and energy efficiency significantly [2]-[6].

With the large number of antennas employed at the BS, one of the key challenges of Massive MIMO is the low-complexity detection in the uplink. Because of the excess degree of freedom in Massive MIMO systems, linear detection algorithms, such as maximum ration combining (MRC), zero forcing (ZF) and minimum mean square error (MMSE), can achieve nearoptimal performance [4]. ZF and MMSE generally perform much better than MRC, but involves matrix inversion which is computationally expensive and non-preferable in hardware implementation [7], which is not taken into consideration in previous works, e.g., [8] and [9].

Recently several papers have worked on the simplification of MMSE detection in the uplink to avoid the inversion of large matrices [10]-[14]. In [10], an approximate matrix approximation based on Neumann series expansion is proposed, which is shown to reduce the complexity substantially, however, suffering from remarkable performance loss. Richardson method is used in [11] to replace the matrix inversion which help to reduce the complexity by an order. In [12], conjugate gradient (CG) method based detection algorithm is proposed. However, Richardson and CG method have many division operations involved and converge slowly. The Gauss-Seidel based approach in [13] performs well with limited number of iterations but not suitable for parallel implementation since an internal iteration is involved. In [14], a near-optimal detection based on joint steepest descent and Jacobi method (referred to as OJA here) is devised to speed up the convergence and enable FPGA implementation.

In this work, we adopt Landweber method to design lowcomplexity uplink detection algorithms. Landweber method uses a series of matrix polynomials and is widely used in solving ill-posed problems [15]. It is stable but converges slowly. In order to reduce the uplink detection complexity and increase the convergence rate, we optimize the relax factor and propose improved Landweber Method with optimal relax factor (ILM-O) algorithm. We also use detection matrices with limited order to approximate those with high order and propose reduced order Landweber Method (ROLM) algorithm. The convergence and the complexity analysis are provided. Numerical results show the proposed algorithms outperform OJA in [14] when the system scales up.

The rest of this paper is organized as follows. The system model is described in Section 2. In Section 3, the proposed linear detection schemes based on Landweber Method are introduced. In Section 4, the performance analysis is provided. Numerical results are given in Section 5 and conclusions are drawn in Section 6.

Notation: vectors and matrices are represented in bold lowercase and capital letters, respectively; $(\bullet)^{\mathrm{T}},(\bullet)^{\mathrm{H}}, \operatorname{Tr}(\bullet)$ denote the transpose and Hermitian transpose, trace of a matrix, respectively; $\mathcal{C N}(\theta, \Sigma)$ denotes the circular symmetric complex Gaussian distribution with mean $\theta$ and covariance $\Sigma$; $\mathbf{I}_{K}$ denotes the $K$ by $K$ identity matrix; $\operatorname{diag}\left\{a_{1}, \cdots, a_{K}\right\}$ denotes a $K$ by $K$ diagonal matrix with diagonal entries given by $a_{1}, \cdots, a_{K} ; \mathbb{E}(\bullet)$ denotes the expectation operation; $\rho_{\mathrm{r}}(\mathbf{A}$ denotes the spectrum radius of $\mathbf{A} ;(a-b)^{+} \triangleq \max \{a-b, 0\}$; $\mathbf{A}^{\dagger}$ denotes the Moore-Penrose pseudo-inverse of $\mathbf{A}$.

\section{SySTEM MOdEL}

Consider the uplink of a multi-user single cell massive MIMO system, where the BS is equipped with $N$ antennas to serve $K$ single-antenna users $(N \gg K)$.

The uplink received signal vector at the BS is given by

$$
\mathbf{y}=\mathbf{H s}+\mathbf{n},
$$


where $\mathbf{s}=\left[s_{1}, \ldots, s_{K}\right] \sim \mathcal{C N}\left(\mathbf{0}, \mathbf{I}_{K}\right)$ with $s_{k}$ being the transmitted signal of the $k$-th user; $\mathbf{n} \sim \mathcal{C N}\left(\mathbf{0}, \sigma_{n}^{2} \mathbf{I}_{N}\right)$ is the noise at the BS; $\mathbf{H} \in \mathbb{C}^{N \times K}$ denotes the the Rayleigh fading channel matrix, the elements of which are independently and identically distributed (i.i.d) with zero mean and unit variance.

In order to detect the transmitted signal vector $\mathbf{s}$ with low computational complexity, we consider linear detection, i.e., the estimate of $\mathbf{s}$ is computed as

$$
\hat{\mathbf{s}}=\mathbf{W y},
$$

where $\mathbf{W} \in \mathbb{C}^{K \times N}$ is the detection matrix.

Existing linear detection method, such as MRC with

$$
\mathbf{W}_{\mathrm{MRC}}=\mathbf{H}^{\mathrm{H}},
$$

ZF with

$$
\mathbf{W}_{\mathrm{ZF}}=\left(\mathbf{H}^{\mathrm{H}} \mathbf{H}\right)^{-1} \mathbf{H}^{\mathrm{H}}
$$

and MMSE with

$$
\mathbf{W}_{\text {MMSE }}=\left(\mathbf{H}^{\mathrm{H}} \mathbf{H}+\sigma_{n}^{2} \mathbf{I}_{K}\right)^{-1} \mathbf{H}^{\mathrm{H}}
$$

are shown to approach the optimal scheme when the system scale becomes large. In practice, however, MRC suffers from significant performance loss while ZF and MMSE involve inversions of large dimensional matrices which is not preferable in hardware implementation.

In this work, we will design low-complexity uplink detection algorithms based on Landweber Method without involvement of matrix inversion.

\section{LiNEAR DETECTION BASED ON LANDWEBER METHOD}

Denote $\mathrm{y}_{\mathrm{a}}=\mathbf{H s}$ as the accurate received signal vector without noise. Assuming $\mathbf{H}$ and $\mathbf{y}$ are perfectly known, we observe from (1) that

$$
\mathbb{E}\left\|\mathbf{y}-\mathbf{y}_{\mathrm{a}}\right\|_{2}^{2}=N \sigma_{n}^{2}
$$

In order to obtain the optimal estimate of $\mathbf{s}$ from the noisepolluted vector $\mathbf{y}$, (2) forms a so called linear ill-posed problem [15] $]^{1}$, which can be solved by Landweber Method.

Landweber Method uses $\mathbf{W}_{\mathrm{L}} \mathbf{y}$ as the estimate of $\mathbf{s}$ where

$$
\mathbf{W}_{\mathrm{L}} \triangleq a \sum_{j=0}^{T}\left(\mathbf{I}_{K}-a \mathbf{H}^{\mathrm{H}} \mathbf{H}\right)^{j} \mathbf{H}^{\mathrm{H}}
$$

in which

$$
0<a<\frac{1}{\rho_{\mathrm{r}}\left(\mathbf{H}^{\mathrm{H}} \mathbf{H}\right)}
$$

is a relax factor to control the convergence, and $T$ is a terminate factor to balance the estimation accuracy and the computational complexity. The application of Landweber method is usually based on iterations. The expression for the $t$-th iteration is given by

$$
\hat{\mathbf{s}}_{t}=\hat{\mathbf{s}}_{t-1}+a \mathbf{H}^{\mathrm{H}}\left(\mathbf{y}-\mathbf{H} \hat{\mathbf{s}}_{t-1}\right),
$$

\footnotetext{
${ }^{1}$ It can also be approximately well-posed under high SNR.
}

with the initial estimate given by $\hat{\mathbf{s}}_{0}=a \mathbf{H}^{\mathrm{H}} \mathbf{y}$. Lanweber method is stable and works well with severe noise. However, it converges slowly and consequently produces processing delay.

In the rest of this section, we will devise two low-complexity detection algorithms based on Landweber Method with optimized relax factor.

\section{A. Improved Landweber Method with Optimized Relax Factor}

According to [15], Landweber Method can be accelerated using a different type of iteration by taking advantage of the special structure of (5). The $(t+1)$-th iteration has the following expression:

$$
\begin{aligned}
\mathbf{W}_{t+1} & =\left(2 \mathbf{I}_{K}-\mathbf{W}_{t} \mathbf{H}\right) \mathbf{W}_{t}, \\
\hat{\mathbf{s}}_{t+1} & =\mathbf{W}_{t+1} \mathbf{y} .
\end{aligned}
$$

After $T$ iterations, the above achieves the same results as (6) with $2^{T}-1$ iterations. Therefore, the convergence rate is greatly improved. However, the optimal choice of $a$ in (5) remains unknown.

From (5), it can be seen that the computational complexity of the detection is related to the spectrum radius of $\mathbf{A}_{a} \triangleq$ $\mathbf{I}_{K}-a \mathbf{H}^{\mathrm{H}} \mathbf{H}$. The matrix polynomial in (5) converges fast with small value of $\rho_{\mathrm{r}}\left(\mathbf{A}_{a}\right)$. Therefore, the optimal $a$ can be achieved by solving the following problem

$$
\begin{aligned}
& a^{*}=\arg \min \rho_{\mathrm{r}}\left(\mathbf{A}_{a}\right) \\
& \text { s.t. } 0<a<\frac{1}{\rho_{\mathrm{r}}\left(\mathbf{H}^{\mathrm{H}} \mathbf{H}\right)} .
\end{aligned}
$$

Proposition 1. Given the assumption in Section 2, the solution to $(8)$ is given by

$$
a^{*}=\frac{1}{N+K} .
$$

Proof: According to [16], in massive MIMO systems when $K$ and $N$ becomes large with a fixed ratio $\eta=\frac{K}{N}$, the eigenvalue distribution of $\frac{1}{N} \mathbf{H}^{\mathrm{H}} \mathbf{H}$ converges to

$$
f_{\frac{1}{N} \mathbf{H}^{\mathrm{H}} \mathbf{H}}(z)=\left(1-\eta^{-1}\right)^{+} \delta(z)+\frac{\sqrt{\left(z-z_{1}\right)^{+}\left(z_{2}-z\right)^{+}}}{2 \pi \eta z},
$$

where $z_{1}=(1-\sqrt{\eta})^{2}$ and $z_{2}=(1+\sqrt{\eta})^{2}$.

Consider $\eta<1$ which is the prevailing scenario. Then when $K$ and $N$ are large, it is easy to achieve

$$
\left.\rho_{\mathrm{r}}\left(\mathbf{A}_{a}\right) \approx \max \left\{\left|1-a N z_{2}\right|, \mid 1-a N z_{1}\right) \mid\right\} .
$$

It is apparent that $z_{1} \leq z_{2}$. Therefore, we can divide the set of feasible $a$ into the following three cases:

i) When $a \leq \frac{1}{N z_{2}}, \rho_{\mathrm{r}}\left(\mathbf{A}_{a}\right)=1-a N z_{1}$. Therefore, solving (8) gives $a^{*}=\frac{1}{N z_{2}}$ and $\min \left\{\rho_{\mathrm{r}}\left(\mathbf{A}_{a}\right)\right\}=\frac{z_{2}-z_{1}}{z_{2}}$;

ii) When $\frac{1}{N z_{2}}<a \leq \frac{1}{N z_{1}}$, there are two cases: either $a N z_{2}-1>1-a N z_{1}$ or the opposite. Both cases lead to the solution of $a^{*}=\frac{2}{N z_{1}+N z_{2}}$ and $\min \left\{\rho_{\mathrm{r}}\left(\mathbf{A}_{a}\right)\right\}=\frac{z_{2}-z_{1}}{z_{1}+z_{2}}$;

iii) When $a>\frac{1}{N z_{1}}, \rho_{\mathrm{r}}\left(\mathbf{A}_{a}\right)=a N z_{2}-1$. Therefore, solving (8) gives $a^{*}=\frac{1}{N z_{1}}$ and $\min \left\{\rho_{\mathrm{r}}(\mathbf{A})\right\}=\frac{z_{2}-z_{1}}{z_{1}}$.

Comparing the minimum spectrum radius of $\mathbf{A}_{a}$ for the above three cases, $\frac{z_{2}-z_{1}}{z_{1}+z_{2}}$ is the smallest. Therefore, the optimal choice of $a$ is $a^{*}=\frac{2}{N z_{1}+N z_{2}}=\frac{1}{N+K}$. 
TABLE I

IMPROVED LANDWEBER METHOD WITH OPTIMIZED RELAX FACTOR $a$ (ILM-O).

\begin{tabular}{cl}
\hline \hline Steps & Operations \\
\hline Input: & $\mathbf{H}, \mathbf{y}, T$ \\
1 & $a=\frac{1}{N+K} ;$ \\
2 & Let $t=0, \mathbf{W}_{t}=a \mathbf{H}^{\mathrm{H}} ;$ \\
3 & while $t<T$ \\
& $3.1 \quad \mathbf{W}_{t+1}=\left(2 \mathbf{I}_{K}-\mathbf{W}_{t} \mathbf{H}\right) \mathbf{W}_{t} ;$ \\
& $3.2 \quad t \leftarrow t+1 ;$ \\
& end \\
4 & $\hat{\mathbf{s}}_{T}=\mathbf{W}_{T} \mathbf{y} ;$ \\
5 & Output $\hat{\mathbf{s}}_{T}$. \\
\hline
\end{tabular}

The algorithm of the improved Landweber Method with optimized relax factor is referred to as ILM-O, the details of which is summarized in Table I.

\section{B. Reduced Order Landweber Method}

IML-O converges very fast, but it involves matrix product which has comparatively higher complexity. In order to reduce the complexity and enable fast convergence of (5), we introduce coefficients to the matrix polynomials in (5) and set up an optimization problem to reduce the order of $\mathbf{W}_{\mathrm{L}}$.

To be specific, we try to use $\mathbf{W}_{\mathrm{L}}$ of order $T$ to approximate that of order $L$ with $T \ll L$ by solving the following problem:

$$
\mathbf{b}^{*}=\arg \min _{\mathbf{b} \in \mathbb{C}^{T+1}} \mathbb{E}\left\|a \sum_{j=0}^{T} b_{j} \mathbf{A}_{a}^{j} \mathbf{H}^{\mathrm{H}} \mathbf{y}-a \sum_{i=0}^{L} \mathbf{A}_{a}^{i} \mathbf{H}^{\mathrm{H}} \mathbf{y}\right\|_{2}^{2} .
$$

where $\mathbf{b}=\left[b_{0}, \ldots, b_{T}\right]^{\mathrm{T}}$.

The above optimization problem is convex over $\mathbf{b}$. The optimal value of $\mathbf{b}$ is given in Proposition 2 .

Proposition 2. The optimal value of $\mathbf{b}$ is give by

$$
\mathbf{b}^{*}=\mathbf{G}^{\dagger} \mathbf{r}
$$

where

$$
\begin{aligned}
& \mathbf{r}=\left[\begin{array}{c}
\sum_{i=0}^{L} \mathbb{E} \operatorname{Tr}\left[\mathbf{A}_{a}^{i} \mathbf{B}\right] \\
\vdots \\
\sum_{i=0}^{L} \mathbb{E} \operatorname{Tr}\left[\mathbf{A}_{a}^{i+T} \mathbf{B}\right]
\end{array}\right], \\
& \mathbf{G}=\left[\begin{array}{ccc}
\mathbb{E} \operatorname{Tr}\left[\mathbf{A}_{a}^{0} \mathbf{B}\right] & \cdots & \mathbb{E} \operatorname{Tr}\left[\mathbf{A}_{a}^{T} \mathbf{B}\right] \\
\vdots & \ddots & \vdots \\
\mathbb{E} \operatorname{Tr}\left[\mathbf{A}_{a}^{T} \mathbf{B}\right] & \cdots & \mathbb{E} \operatorname{Tr}\left[\mathbf{A}_{a}^{T+T} \mathbf{B}\right]
\end{array}\right],
\end{aligned}
$$

and

$$
\mathbf{B}=\mathbf{H}^{\mathrm{H}}\left(\mathbf{H} \mathbf{H}^{\mathrm{H}}+\sigma_{n}^{2} \mathbf{I}_{N}\right) \mathbf{H}
$$

The mathematical expectations in (14) are calculated as

$$
\mathbb{E} \operatorname{Tr}\left[\mathbf{A}_{a}^{i} \mathbf{B}\right]=K \int(1-a z)^{i}\left(z^{2}+z \sigma_{n}^{2}\right) \frac{1}{N} f_{\frac{1}{N} \mathbf{H}^{\mathrm{H}} \mathbf{H}}\left(\frac{z}{N}\right) \mathrm{d} z .
$$

TABLE II

REDUCED ORDER LANDWEBER METHOD (ROLM).

\begin{tabular}{cl}
\hline \hline Steps & Operations \\
\hline Input: & $\mathbf{H}, \mathbf{y}, T$. \\
1 & $a=\frac{1}{N+K}$, compute $\mathbf{b}$ according to (13); \\
2 & Let $t=0, \mathbf{C}=\mathbf{H}^{\mathrm{H}} \mathbf{H}, \hat{\mathbf{s}}_{t}=a b_{t} \mathbf{H}^{\mathrm{H}} \mathbf{y} ;$ \\
3 & while $t<T$ \\
& $3.1 \quad \hat{\mathbf{s}}_{t+1}=\left(1+a b_{t}\right) \hat{\mathbf{s}}_{t}-a^{2} b_{t} \mathbf{C} \hat{\mathbf{s}}_{t} ;$ \\
& $3.3 \quad t \leftarrow t+1 ;$ \\
& end \\
4 & Output $\hat{\mathbf{s}}_{T}$.
\end{tabular}

Proof: Taking the first order derivative of (12) with respect to $b_{j}$ gives

$$
\sum_{i=0}^{T} b_{i} \mathbb{E} \operatorname{Tr}\left[\mathbf{A}_{a}^{i+j} \mathbf{B}\right]-\sum_{i=0}^{L} \mathbb{E} \operatorname{Tr}\left[\mathbf{A}_{a}^{j+i} \mathbf{B}\right]=0, \quad j=0, \ldots, T .
$$

Solving the above equation array gives (13).

Denote $\mathbf{C}=\mathbf{H}^{\mathrm{H}} \mathbf{H}$, with the coefficient $\mathbf{b}$, the $(t+1)$-th iteration becomes

$$
\begin{aligned}
\hat{\mathbf{s}}_{t+1} & =\hat{\mathbf{s}}_{t}+a b_{t}\left(\mathbf{I}_{K}-a \mathbf{C}\right) \hat{\mathbf{s}}_{t} \\
& =\left(1+a b_{t}\right) \hat{\mathbf{s}}_{t}-a^{2} b_{t} \mathbf{C} \hat{\mathbf{s}}_{t} .
\end{aligned}
$$

The reduced order Landweber method is summarized in Table II, which is referred to as ROLM. Note that the proposed method is different from that in [17] where the coefficients are optimized to minimize the mean squared error of the channel estimator, while in this work the coefficients are optimized to reduce the order of the polynomials so that the iteration of Landweber Method still holds. Moreover, the expectations in the optimization problems in this work are different from [17] and calculated in different ways.

\section{Performance AnAlysis}

In this section, we carry out performance analysis of the proposed linear detection algorithms in terms of convergence and computational complexity.

According to Proposition 1, the spectrum radius is given by

$$
\min \rho_{\mathrm{r}}\left(\mathbf{A}_{a}\right)=\frac{2 \sqrt{\eta}}{1+\eta} .
$$

Since $0<\eta<1,1+\eta>2 \sqrt{\eta}$, we have $0<\rho_{\mathrm{r}}\left(\mathbf{A}_{a}\right)<1$. Therefore, the convergence of (5) is fulfilled and the same for ROLM and ILM-O.

To analyze the computational complexity, we use the total number of floating-point operations (FLOPs) involved in the algorithms. Each real-valued multiplication or addition counts for 1 FLOP, while one complex-valued multiplication and addition counts for 6 FLOPs and 2 FLOPs, respectively. The multiplication of an $N \times K$ and a $K \times M$ complex matrix requires $N M(8 K-2)$ FLOPs.

To capture the dominant terms, we only count matrix-matrix and matrix-vector products in the following analysis. For ILM$\mathrm{O}$, it involves two matrix multiplications in each iteration and 
TABLE III

COMPLEXITY COMPARISON OF THE PROPOSED ILM-O, ROLM ALGORITHM AND OJA IN [14].

\begin{tabular}{cl}
\hline \hline Algorithms & Number of FLOPs \\
\hline ILM-O & $\left(4 T K^{2}-2 T K+2 K\right) N-2 T K^{2}-2 K$ \\
ROLM & $2\left(K^{2}+K\right) N+(2 T-2) K^{2}-(2 T+2) K$ \\
OJA & $2\left(K^{2}+K\right) N+(2 T+2) K^{2}-(2 T+6) K$ \\
\hline
\end{tabular}

one matrix-vector multiplication in step 4 , which counts for a total of $\left(4 T K^{2}-2 T K+2 K\right) N-2 T K^{2}-2 K$ FLOPs. For ROLM, since the optimal value of $\mathbf{b}$ is calculated only once, the computational cost can be omitted. In ROLM, one matrix and one matrix-vector multiplication are required in step 2 and 1 matrix-vector product is involved in each iteration of step 3 , which counts for a total of $2\left(K^{2}+K\right) N+(2 T-2) K^{2}-$ $(2 T+2) K$.

The complexity comparison of ILM-O, ROLM and OJA in [14] is summarized in Table III. It can be seen from Table III that the complexity of ROLM is comparable to OJA in [14], while ILM-O has the largest computational cost in most cases.

\section{Numerical Results}

In this section, we carry out Monte Carlo simulations to evaluate the proposed ILM-O and ROLM in terms of bit error rate (BER). We also compare the proposed algorithms with the existing algorithm, i.e., MMSE, MRC and OJA in [14] which is shown to outperform the other existing schemes. Comprehensive evaluation and analysis remain for future work.

In all the simulations, the number of antennas at the BS is $N=100$, while the number of users is $K=20,40$. In terms of modulation, 64-ary Quadrature Amplitude Modulation (64QAM) is considered according to [14] with normalized power. The signal to noise ratio (SNR) is defined as $\frac{1}{\sigma_{n}^{2}}$.

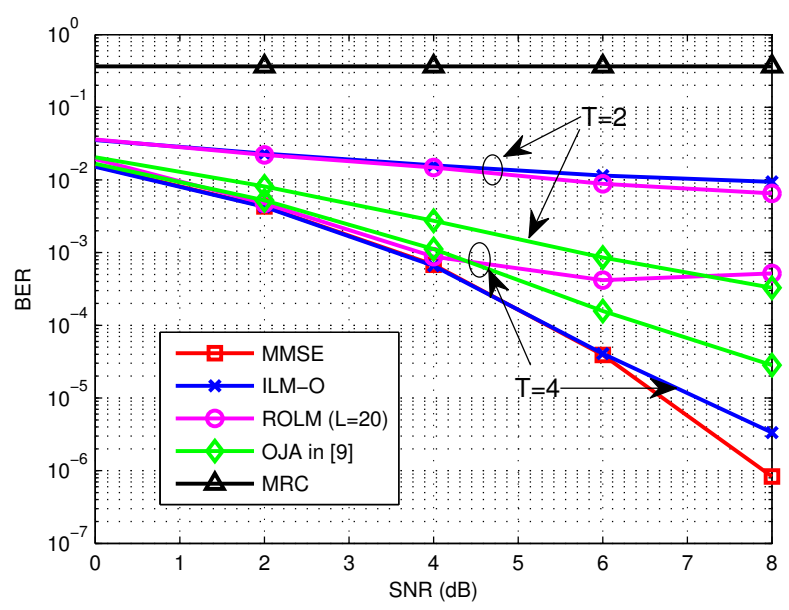

Fig. 1. Comparison between OJA in [14] and the proposed ILM-O,ROLM algorithms, where $N=100, K=20$.

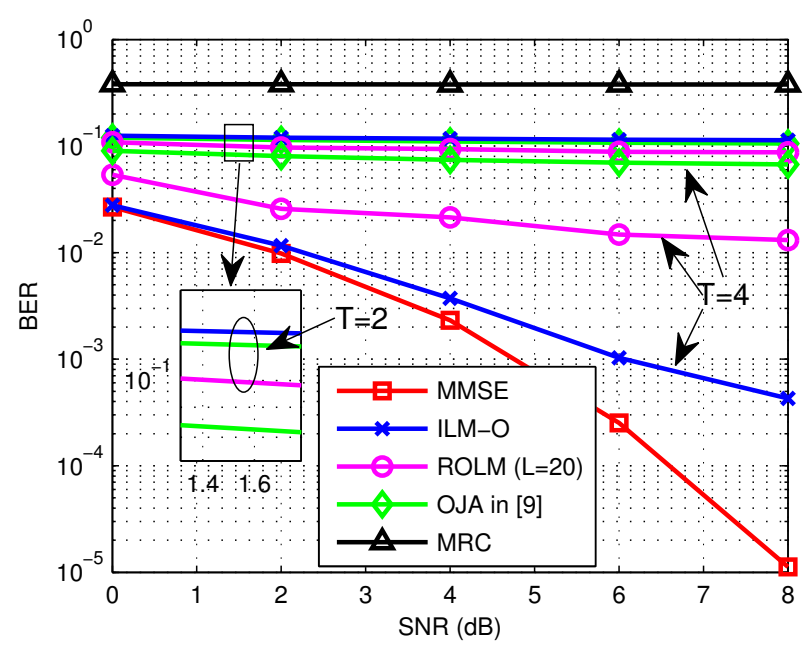

Fig. 2. Comparison between OJA in [14] and the proposed ILM-O,ROLM algorithms, where $N=100, K=40$

Figure 1 depicts the performance comparison of OJA in [14], ILM-O and ROLM for $T=2, T=4$ when $K=20$. As can be seen in Figure 1, with increased SNR the BERs of all three algorithms decrease. When $T=2$, OJA in [14] performs better the ILM-O and ROLM. However,when $T=4$, the proposed ILM-O outperforms OJA and ROLM significantly with a gain over $2 \mathrm{~dB}$ in high SNR region. Although OJA works better than ROLM, it involves division operations, which is not preferable in hardware implementation. The complexity of OJA is also slightly higher than ROLM. It can be observed that ROLM reaches a error floor due to the fact that the approximation made in ROLM dominates the BER performance over noise in the high SNR region. Considering most channel codes work well with a BER range of $10^{-1}$ to $10^{-2}$, all three algorithms show good performance.

As is shown in Figure 2, when $K=40$ the proposed algorithms show significant advantages over OJA. For $T=2$, ROLM performs better than OJA and ILM-O, due to the optimized polynomial coefficients which enable ROLM to approximate the performance of higher order $\mathbf{W}_{\mathrm{L}}$. When $T=4$, both the proposed algorithms outperform OJA significantly. This is because Landweber Method is very stable and work well in different scenarios. Compared with ROLM, ILM-O performs better but with comparably heavier computation cost.

\section{CONCLUSions}

In this paper, we have proposed two uplink detection algorithms based on Landweber Method for Massive MIMO systems. We have derived the optimal relax factor for the improved Landweber Method and proposed ILM-O algorithms. In order to reduce the computational complexity, reduced order Landweber Method (ROLM) is proposed by approximate high order detection matrix within limited iterations. Convergence and computational complexity are analyzed. Numerical results show that the proposed ILM-O and ROLM algorithms work 
well and outperform existing OJA algorithm in [14] when the system scales up.

\section{ACKNOWLEDGEMENT}

This work was supported in part by Research Foundation for Advanced Talents of Jiangsu University (No.16JDG065), National Natural Science Foundation of China (No.61502210), China Postdoctoral Science Foundation (No.2015M570484).

\section{REFERENCES}

[1] Emre Telatar, "Capacity of Multi-antenna Gaussian Channels," European transactions on telecommunications, , no. 2, pp. 1-28, Nov. 1999.

[2] Thomas L Marzetta, "Noncooperative Cellular Wireless with Unlimited Numbers of Base Station Antennas," IEEE Transactions on Wireless Communications, vol. 9, no. 11, pp. 3590-3600, November 2010.

[3] $\mathrm{Lu} \mathrm{Lu}$, Geoffrey Li, A. Lee Swindlehurst, Alexei Ashikhmin, and Rui Zhang, "An Overview of Massive MIMO: Benefits and Challenges," IEEE Journal of Selected Topics in Signal Processing, vol. 8, no. 5, pp. 742-758, 2014.

[4] Fredrik Rusek, Daniel Persson, E. G. Larsson, T. L. Marzetta, and Fredrik Tufvesson, "Scaling Up MIMO: Opportunities and Challenges with Very Large Arrays," IEEE Signal Processing Magazine, vol. 30, no. 1, pp. 40-60, January 2013.

[5] Erik Larsson, Ove Edfors, Fredrik Tufvesson, and Thomas Marzetta, "Massive MIMO for next generation wireless systems," IEEE Coтmunications Magazine, vol. 52, no. 2, pp. 186-195, Feb. 2014.

[6] Wence Zhang, Hong Ren, Cunhua Pan, Ming Chen, R.C. de Lamare, Bo Du, and Jianxin Dai, "Large-scale antenna systems with ul/dl hardware mismatch: Achievable rates analysis and calibration," IEEE Transactions on Communications, vol. 63, no. 4, pp. 1216-1229, April 2015.

[7] Hemanth Prabhu, Ove Edfors, Joachim Rodrigues, Liang Liu, and Fredrik Rusek, "Hardware efficient approximative matrix inversion for linear pre-coding in massive MIMO," in 2014 IEEE International Symposium on Circuits and Systems (ISCAS), June 2014, pp. 1700-1703.

[8] A. Elghariani and M. Zoltowski, "Low complexity detection algorithms in large-scale mimo systems," IEEE Transactions on Wireless Communications, vol. 15, no. 3, pp. 1689-1702, March 2016.

[9] J. W. Choi, B. Lee, B. Shim, and I. Kang, "Low complexity detection and precoding for massive mimo systems," in 2013 IEEE Wireless Communications and Networking Conference (WCNC), April 2013, pp. 2857-2861.

[10] M. Wu, B. Yin, G. Wang, C. Dick, J. R. Cavallaro, and C. Studer, "Large-scale mimo detection for 3gpp lte: Algorithms and fpga implementations," IEEE Journal of Selected Topics in Signal Processing, vol. 8, no. 5, pp. 916-929, Oct 2014.

[11] X. Gao, L. Dai, Y. Ma, and Z. Wang, "Low-complexity near-optimal signal detection for uplink large-scale MIMO systems," Electronics Letters, vol. 50, no. 18, pp. 1326-1328, August 2014.

[12] B. Yin, M. Wu, J. R. Cavallaro, and C. Studer, "Conjugate gradientbased soft-output detection and precoding in massive MIMO systems," in 2014 IEEE Global Communications Conference, Dec 2014, pp. 36963701.

[13] L. Dai, X. Gao, X. Su, S. Han, C. L. I, and Z. Wang, "Low-Complexity Soft-Output Signal Detection Based on Gauss-Seidel Method for Uplink Multiuser Large-Scale MIMO Systems," IEEE Transactions on Vehicular Technology, vol. 64, no. 10, pp. 4839-4845, Oct 2015.

[14] X. Qin, Z. Yan, and G. He, "A Near-Optimal Detection Scheme Based on Joint Steepest Descent and Jacobi Method for Uplink Massive MIMO Systems," IEEE Communications Letters, vol. 20, no. 2, pp. 276-279, Feb 2016.

[15] Jun Zhang, "An Accelerated Landweber Iteration for Solving Ill-Posed Problems," Journal of Mathematics, no. 3, pp. 333-335, 2005.

[16] Antonia Maria Tulino and Sergio Verdú, Random Matrix Theory and Wireless Communications, Now Publishers Inc, Hanover, 2004.

[17] Nafiseh Shariati, Emil Bjornson, Mats Bengtsson, and Merouane Debbah, "Low-Complexity Polynomial Channel Estimation in Large-Scale MIMO with Arbitrary Statistics," IEEE Journal of Selected Topics in Signal Processing, vol. 8, no. 5, pp. 815-830, 2014. 